\title{
LINEAR DIFFERENTIAL EQUATIONS IN DISTRIBUTIONS
}

\author{
LESLIE D. GATES, JR. ${ }^{1}$
}

1. Introduction. The main result of this paper is an extension of the analysis of Laurent Schwartz [1] concerning the primitive of a distribution. We follow the definitions and notation of Schwartz.

In particular, let $D$ denote the linear space of "testing functions" $\phi(x)$ of the real variable $x$. The functions $\phi(x)$ are indefinitely differentiable and vanish identically outside of some bounded interval. Convergence in $D$ is defined by:

Definition 1. The sequence of testing functions $\left\{\phi_{j}\right\}$ converges to the function $\phi \equiv 0\left(\left\{\phi_{j}\right\} \rightarrow 0\right)$ if and only if

(a) $\phi_{j}^{(k)}(x)$ approaches zero uniformly in $x$ (but not necessarily in $k$ ) for all $k$.

(b) Every $\phi_{j}$ vanishes identically outside of a bounded interval common to all.

Let $D^{\prime}$ denote the space of linear continuous functionals (distributions) on $D$. Continuity requires that if the sequence $\left\{\phi_{j}\right\} \rightarrow 0$, the sequence of (real) numbers $T \cdot \phi_{j}$, where $T$ is a distribution, also approaches zero. The linear space $D^{\prime}$ is the dual or conjugate space of $D$. If $f(x)$ is a function Lesbesgue integrable over every finite interval, and

$$
T \cdot \phi=\int_{-\infty}^{\infty} f(x) \phi(x) d x,
$$

we shall say that $T$ corresponds to $f(T \leftrightarrow f)$. Actually the correspondence is to a class of functions identical except on a set of measure zero.

Let $E$ denote the space of real, indefinitely differentiable functions of $x$.

We shall use small Greek letters for testing functions and capital Roman letters for distributions, except when a distribution corresponds to a function, when small Roman letters will be used; i.e., $f \leftrightarrow f(x)$.

The spaces $D$ and $D^{\prime}$ are paired orthogonally in the sense of Lefschetz. It follows from Lefschetz [2], that if $T_{1}, T_{2}, \cdots, T_{m}$ are a linearly independent set of distributions, a linearly independent set

Received by the editors July 20, 1955 and, in revised form, October 24, 1955.

1 This paper is based on a doctoral dissertation written under the direction of Professor H. D. Block and submitted to Iowa State College in July, 1952. The author is indebted to Professor Block for guidance and encouragement.

The author is presently at the U. S. Naval Proving Ground. 
of testing functions $\phi_{1}, \phi_{2}, \cdots, \phi_{m}$ exists such that

(Kronecker symbol).

$$
T_{i} \cdot \phi_{j}=\delta_{i j}
$$

A product of two distributions can always be defined if one of them corresponds to a function in $E$. If $f \leftrightarrow f(x) \in E$, then

for arbitrary $T$.

$$
(f T) \cdot \phi=(T f) \cdot \phi=T \cdot(f \phi)
$$

The derivative of a distribution is defined by

$$
T^{\prime} \cdot \phi=-T \cdot \phi^{\prime}
$$

2. A theorem on linear differential equations in distributions.

Definition 2. The equation

$$
L[T]=p_{0} T^{(n)}+p_{1} T^{(n-1)}+\cdots+P_{n} T=S,
$$

where each $p_{i} \leftrightarrow p_{i}(x) \in E$, and $S$ is a given distribution, is a linear differential equation in distributions of order $n$, with coefficients in $E$. If $S=0$, the equation is homogeneous.

TheOREM 1. $L[T] \cdot \phi=T \cdot \bar{L}[\phi]$, where $\bar{L}$ is the differential operator adjoint to $L$.

Proof.

$$
\begin{aligned}
L[T] \cdot \phi & =\left(\sum_{i=0}^{n} p_{i} T^{(n-i)}\right) \cdot \phi=\sum_{i=0}^{n} T^{(n-i)} \cdot\left(p_{i} \phi\right) \\
& =T \cdot\left(\sum_{i=0}^{n}(-1)^{n-i}\left(p_{i} \phi\right)^{(n-i)}\right)=T \cdot \bar{L}[\phi] .
\end{aligned}
$$

TheOREM 2. The operation defined by $\theta=\bar{L}[\phi]$ maps $D$ onto $D_{\theta}, a$ linear subspace of $D$, continuously in the sense of $D$.

Proof. The linearity of the operator $\bar{L}$ insures that $D_{\theta}$ is a linear subspace of $D$. Also if $\left\{\phi_{j}\right\} \rightarrow 0$, it is easy to see that

$$
\left\{\theta_{j}\right\}=\left\{\bar{L}\left[\phi_{j}\right]\right\}=\left\{\sum_{i=0}^{n}(-1)^{n-i}\left(p_{i} \phi_{j}\right)^{(n-i)}\right\}=\left\{\sum_{i=0}^{k} q_{i} \phi_{j}^{(i)}\right\} \rightarrow 0
$$

where $q_{i} \in E$ for all $i$.

THEOREM 3.

a. If $T_{1}$ and $T_{2}$ are solutions of $L[T]=0$, then $C_{1} T_{1}+C_{2} T_{2}$ is also a solution, where $C_{1}$ and $C_{2}$ are arbitrary constants.

b. If $T_{1}$ and $T_{2}$ are solutions of $L[T]=S$, then $T_{1}-T_{2}$ is a solution of $L[T]=0$. 
c. The totality of solutions of $L[T]=0$ forms a linear subspace of $D^{\prime}$ which is the annihilator of $D_{\theta}$.

Proof. Statements a and b follow immediately from the linearity of the operator $L$. Statement $\mathrm{c}$ follows from a and Theorem 1.

Theorem 4. If (a) the mapping of $D$ onto $D_{\theta}$ defined by $\bar{L}$ is one-toone and has an inverse $\bar{L}^{-1}$ continuous in the sense of $D$, and (b) $T_{1} \cdot \phi=T_{2} \cdot \phi=\cdots=T_{m} \cdot \phi=0$ are necessary and sufficient conditions that $\phi$ be in $D_{\theta}$, then the differential equation

$$
L[T]=S
$$

has a solution which can be written

$$
T \cdot \phi=\sum_{i=1}^{m} C_{i} T_{i} \cdot \phi+S \cdot\left(\bar{L}^{-1}\left[\phi-\sum_{i=1}^{m} a_{i} \psi_{i}\right]\right),
$$

where $a_{i}=T_{i} \cdot \phi$, the $C_{i}$ are arbitrary constants, and the $\psi_{i}$ are a set of $m$ testing functions such that

$$
T_{i} \cdot \psi_{k}=\delta_{i k}, \quad i, k=1,2, \cdots, m .
$$

Proof. The existence of the $\psi_{i}$ is assured by the result quoted from [2]. Since $\bar{L}^{-1}$ is the inverse of a linear operator, it is itself linear and this insures that $T$ as defined is a linear functional. If a sequence $\left\{\phi_{j}\right\} \rightarrow 0$, then the $a_{i j}=T_{i} \cdot \phi_{j} \rightarrow 0$, so that the sequence

$$
\left\{\phi_{i}-\sum_{i=1}^{m} a_{i j} \psi_{i}\right\} \rightarrow 0
$$

Since the operator $\bar{L}^{-1}$ is continuous in the sense of $D$ and $S$ is a distribution

$$
S \cdot\left(\bar{L}^{-1}\left[\phi_{j}-\sum_{i=1}^{m} a_{i j} \psi_{i}\right]\right) \rightarrow 0
$$

and thus $T \cdot \phi_{j} \rightarrow 0$. This shows that $T$ is continuous and thus indeed a distribution.

Now for an arbitrary $\phi \in D, L[T] \cdot \phi=T \cdot \bar{L}[\phi]$. By hypothesis (b), $T_{i} \cdot \bar{L}[\phi]=0$, so that the $a_{i}$ corresponding to $\bar{L}[\phi]$ are zero. Thus we have

$$
\begin{aligned}
L[T] \cdot \phi & =\sum_{i=1}^{m} C_{i} T_{i} \cdot \bar{L}[\phi]+S \cdot \bar{L}^{-1}[\bar{L}[\phi]] \\
& =S \cdot \phi
\end{aligned}
$$


as desired. Also, since for all $j$

$$
\begin{aligned}
T_{j} \cdot\left(\phi-\sum_{i=1}^{m} a_{i} \psi_{i}\right) & =T_{i} \cdot \phi-\sum_{i=1}^{m} a_{i} T_{i} \cdot \psi_{i} \\
& =T_{j} \cdot \phi-a_{j} \\
& =0
\end{aligned}
$$

the testing function $\phi-\sum_{i=1}^{m} a_{i} \psi_{i}$ is in $D_{\theta}$ by hypothesis (b). It is, therefore, a suitable operand for $\bar{L}^{-1}$.

It will be immediately noticed that the sum $\sum_{i+1}^{m} C_{i} T_{i}$ is a linear combination of solutions of the homogeneous equation $L[T]=0$, while the remainder of $T$ corresponds to the "particular integral" of differential equations in functions.

3. Examples. We give first an example where hypothesis (b) fails. Let the function $p(x)$ be defined by

$$
p(x)= \begin{cases}\exp \left(x^{2}-1\right)^{-1}, & |x|<1, \\ 0, & |x| \geqq 1\end{cases}
$$

Consider the differential equation

$$
p T^{\prime}+p^{\prime} T=S, \quad p \leftrightarrow p(x) .
$$

We have

$$
\begin{aligned}
\left(p T^{\prime}+p^{\prime} T\right) \cdot \phi & =T \cdot\left(-(p \phi)^{\prime}+p^{\prime} \phi\right) \\
& =T \cdot\left(-p \phi^{\prime}\right) .
\end{aligned}
$$

The subspace $D_{\theta}$ is composed of testing functions of the form

$$
\theta=\bar{L}[\phi]=-p \phi^{\prime}
$$

for $\phi$ arbitrary in $D$. But it is evident that $\bar{L}$ could not have an inverse since a sequence $\left\{\phi_{j}\right\}$ could fail to converge by reason of diverging supports, and yet the corresponding $\left\{\theta_{j}\right\}$ could converge, since each $\theta_{j}$ vanishes outside of $(-1,1)$. (The support of a testing function is the closure of the set on which it is different from zero.) If

$$
S \cdot \phi=\int_{-\infty}^{\infty} \phi(x) d x
$$

and a sequence $\left\{\phi_{j}\right\}$ is chosen such that

$$
\phi_{j}= \begin{cases}\frac{k}{j} \exp \frac{j^{2}}{x^{2}-j^{2}}, & |x|<j, \\ 0, & |x| \geqq j,\end{cases}
$$


where $k$ satisfies

$$
k \int_{-1}^{1} \exp \left(x^{2}-1\right)^{-1} d x=1
$$

then it can be shown that although

$$
\left\{-p \phi_{j}^{\prime}\right\} \rightarrow 0, \quad S \cdot \phi_{j}=1
$$

for all $j$, so that

$$
T \cdot\left[-p \phi^{\prime}\right]=S \cdot \phi
$$

does not define a distribution, and the differential equation can not have a solution on an interval which includes 1 or -1 .

As an example of a well behaved equation, consider

$$
x T^{\prime}+T=S(x \leftrightarrow f(x) \equiv x),
$$

a special case of an equation whose solution was given by Schwartz [1], for the homogeneous case $S=0$. We have

$$
\left(x T^{\prime}+T\right) \cdot \phi=T \cdot\left(-x \phi^{\prime}\right)=S \cdot \phi .
$$

The corresponding equation in testing functions is

$$
\theta=-x \phi^{\prime} \text {. }
$$

Obviously, $\theta(0)=0$. For all $x<0$, we can define a solution by

$$
-\phi(x)=\int_{-\infty}^{x} \frac{\theta}{x} d x .
$$

Integrating by parts, we get

$$
\begin{aligned}
\phi(x) & =-\theta(x) \ln |x|+\int_{-\infty}^{x} \ln |x| \theta^{\prime}(x) d x \\
& =\bar{L}^{-1}[\theta]
\end{aligned}
$$

and this definition will serve for all $x$. The condition $\phi(\infty)=0$ gives the necessary condition on $\theta$

$$
\int_{-\infty}^{\infty} \ln |x| \theta^{\prime}(x) d x=0
$$

(the condition $\phi(-\infty)=0$ requires that the constant of integration be zero). It can be shown that these conditions on $\theta$ are also sufficient to insure that $\phi$ is a testing function. The first condition is expressed by the "Dirac" distribution 


$$
T_{1} \cdot \phi=\phi(0)_{i}^{j}=0
$$

while the second can be expressed by the "pseudo function $1 / x$ "

$$
T_{2} \cdot \phi=\int_{-\infty}^{\infty} \ln |x| \phi^{\prime}(x) d x=0 .
$$

The entire solution obtainable from Theorem 4 is then

$$
\begin{aligned}
T \cdot \phi=C_{1} T_{1} \cdot \phi & +C_{2} T_{2} \cdot \phi \\
& +S \cdot\left(-\theta(x) \ln |x|+\int_{-\infty}^{x} \ln |x| \theta^{\prime}(x) d x\right)
\end{aligned}
$$

where

$$
\begin{aligned}
\theta & =\phi-a_{1} \psi_{1}-a_{2} \psi_{2}, \\
a_{i} & =T_{i} \cdot \phi, \\
T_{i} \cdot \psi_{j} & =\delta_{i j} .
\end{aligned}
$$

In the above example, take $S=\delta$, the Dirac distribution. Then the particular integral above becomes

$$
\begin{aligned}
\int_{-\infty}^{0} \ln |x| \phi^{\prime}(x) d x & -a_{1} \int_{-\infty}^{0} \ln |x| \psi_{1}^{\prime}(x) d x \\
& -a_{2} \int_{-\infty}^{0} \ln |x| \psi_{2}^{\prime}(x) d x .
\end{aligned}
$$

$\left[\theta(0) \ln 0\right.$ is defined to be $\lim _{x \rightarrow 0} \theta(x) \ln |x|=0$.]

The last two of these terms are multiples of $a_{1}=T_{1} \cdot \phi$ and $a_{2}=T_{2} \cdot \phi$. The first term is the "pseudo function"

$$
f(x)= \begin{cases}1 / x, & x<0, \\ 0, & x \geqq 0 .\end{cases}
$$

Thus the complete solution of

$$
x T^{\prime}+T=\delta
$$

is given by

$$
\begin{aligned}
T \cdot \phi= & C_{1} \phi(0)+C_{2} \int_{-\infty}^{\infty} \ln |x| \phi^{\prime}(x) d x \\
& +\int_{-\infty}^{0} \ln |x| \phi^{\prime}(x) d x .
\end{aligned}
$$


An analysis equivalent to the above for the equation

$$
x T^{\prime}-a T=S
$$

for $-\infty<a<\infty$ as well as for a few other equations is given by Gates in $[3]$.

This example illustrates the fact that the solution to a differential equation in distributions is equivalent to a solution of the adjoint equation in testing functions, together with a characterization of the subspace $\bar{L}[\phi]$ in terms of its annihilator.

4. Some remaining questions. Additional questions which the author would like to see answered concern two aspects of the general problem.

First, are the hypotheses of Theorem 4 sufficient to insure that a complete solution has been obtained? If it is true for the space $D$ and its dual $D^{\prime}$ that the annihilators of subspaces have the reflexive property (that is, that $D_{\theta} \subset D$ is the annihilator of $D_{\theta}^{\prime} \subset D^{\prime}$ implies that $D_{\theta}^{\prime}$ is the annihilator of $D_{\theta}$ ), then the question is answered in the affirmative. For the hypothesis ' $T_{1} \cdot \phi=T_{2} \cdot \phi=\cdots=T_{m} \cdot \phi=0$ is equivalent to $\phi \in D_{\theta}$ ' would then be sufficient to insure that the subspace spanned by the $T_{i}, i=1,2, \cdots, m$, is the annihilator of $D_{\theta}$ and therefore the complete subspace of solutions of the homogeneous equation $L[T]=0$.

Secondly, it will have been observed that the number of independent solutions is not necessarily equal to the order of the equation. This fact is related to the occurrence of zeros in the coefficients of the equation. Also, the example of the equation where no solution existed was characterized by an "essential" singularity in the leading coefficient. It would be interesting to have a theory which related the existence and number of independent solutions to the order of the zeros in the several coefficients. Such a theory would undoubtedly be related to the corresponding theory for ordinary equations.

\section{REFERENCES}

1. L. Schwartz, Theorie des distributions, vol. 1, Paris, 1950. (Coll. A.S.I., No. 1090.)

2. S. Lefschetz, Algebraic topology, Amer. Math. Soc. Colloquium Publications, vol. 27, New York, 1942, p. 77.

3. L. D. Gates, Jr., Differential equations in the distributions of Schwartz, Doctoral Thesis, Iowa State College, 1952.

Iowa State College 